\title{
Magnetization Dynamics and Coherent Spin Manipulation of a Propeller Gd(III) Complex with the Smallest Helicene Ligand
}

\author{
Gabriela Handzlik, ${ }^{\dagger}$ Michał Magott, ${ }^{\dagger}$ Mirosław Arczyński, Alena M. Sheveleva, Floriana Tuna, \\ Marcin Sarewicz, Artur Osyczka, Michał Rams, Veacheslav Vieru, Liviu F. Chibotaru, \\ and Dawid Pinkowicz*
}

Cite This: J. Phys. Chem. Lett. 2020, 11, 1508-1515

Read Online

ABSTRACT: A homoleptic gadolinium(III) complex with the smallest helicene-type ligand, 1,10-phenanthroline- $N, N^{\prime}$-dioxide (phendo) $\left[\mathrm{Gd}(\text { phendo) })_{4}\right]\left(\mathrm{NO}_{3}\right)_{3} \cdot x \mathrm{MeOH}$ (phendo = 1,10-phenanthroline- $N, N^{\prime}$-dioxide, $\mathrm{MeOH}=$ methanol), shows slow relaxation of the magnetization characteristic for Single Ion Magnets (SIM), despite negligible magnetic anisotropy, confirmed by ab initio calculations. Solid state dilution magnetic and EPR studies reveal that the magnetization dynamics of the $\left[\mathrm{Gd}(\text { phendo })_{4}\right]^{3+}$ cation is controlled mainly by a Raman process. Pulsed EPR experiments demonstrate long phase memory times (up to $2.7 \mu \mathrm{s}$ at $5 \mathrm{~K}$ ), enabling the detection of Rabi oscillations at $20 \mathrm{~K}$, which confirms coherent control of its spin state.

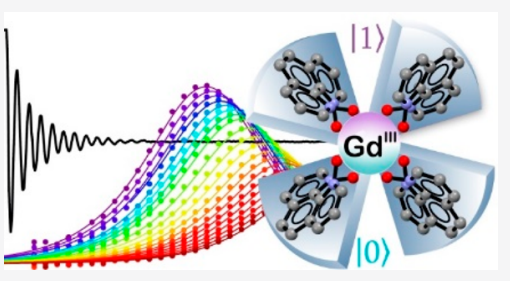

ingle molecule magnets (SMMs) enjoy renewed interest in $\checkmark$ the field of molecular magnetism ${ }^{1,2}$ due to a rapid increase of the magnetization blocking temperatures approaching the boiling point of nitrogen ${ }^{3-5}$ and their implementation as molecular qubits. Lanthanide complexes turned out to be much better candidates for high-temperature SMMs than the transition metals, ${ }^{6}$ even though the latter can easily form highspin coordination clusters. ${ }^{7}$

Rational tuning of the magnetic anisotropy by employing a proper ligand field was found to be the most efficient strategy toward higher spin reversal barriers in the design of SMMs based on single lanthanide ions (single ion magnets, SIMs). ${ }^{8}$ High magnetic anisotropy is necessary for achieving high energy barriers for spin reversal, but not sufficient to get an excellent SMM because the spin-lattice (direct, Raman) relaxation processes or the quantum tunneling of magnetization (QTM) may significantly affect the blocking temperature. $^{9-12}$ In particular, Raman relaxation was recently found to be the limiting process below the blocking temperature of the record-breaking SIMs. ${ }^{3,4,13}$

Single ion magnets displaying a wide magnetic hysteresis are potential candidates for data storage. ${ }^{14}$ On the other hand, molecular nanomagnets with no magnetic hysteresis, but with sufficiently long quantum coherence times $\left(T_{\mathrm{m}}\right)$, are good candidates for spin qubits and quantum information processing. ${ }^{15}$ This coherence time (called also phase memory time) determines the ability of a spin qubit to remain in the superposition of two states and to resist any uncontrolled interaction with its environment, thus, determining its utility in quantum technologies. ${ }^{16}$ Lanthanide spin qubits are especially interesting, as they enable realization of multiple addressable qubits within a single molecule ${ }^{17}$ or encoding an electron qubit using a nuclear qudit. ${ }^{18}$ However, any potential application of lanthanide-based qubits is currently hindered by their coherence times, which are usually shorter by an order of magnitude as compared to $3 d$ metal complexes. ${ }^{19}$ Therefore, new studies in this area are required to overcome this drawback.

There are only several examples of slow magnetic relaxation for gadolinium(III) complexes. ${ }^{20-26}$ The main reason is the magnetic isotropy of $\mathrm{Gd}^{\mathrm{III}}$, which renders it unuseful for the design of SIMs. Due to the lack of an intrinsic magnetic anisotropy, any slow magnetic relaxation for $\mathrm{Gd}^{\mathrm{III}}$ complexes must stem from either (i) weak anisotropy ${ }^{21}$ caused by orbital nondegeneracy of the ground term ${ }^{8} S$ or (ii) low rate of the relaxation processes other than Orbach, which become dominant when the energy difference between all $m_{\mathrm{J}}$ states of the $\mathrm{Gd}^{\mathrm{III}}$ ion is very small. Hence, $\mathrm{Gd}^{\mathrm{III}}$-based SIMs give the perfect opportunity to study these processes. However, for the $\mathrm{Gd}^{\mathrm{III}}$ complexes studied so far, the slow magnetic relaxation is often attributed to the phonon bottleneck effect, and the spinlattice relaxation was postulated to appear only under special circumstances, that is, in the presence of $\mathrm{Gd}-\mathrm{Pt}$ bonds. ${ }^{24}$

In this work we present a 4-fold propeller-shaped $\mathrm{Gd}^{\mathrm{III}}$ complex with the smallest helicene-type ligand $N, N^{\prime}$ dioxophenanthroline (phendo $)^{27}\left[\mathrm{Gd}(\text { phendo })_{4}\right]\left(\mathrm{NO}_{3}\right)_{3}$. $x \mathrm{MeOH}$ (1) with a slow relaxation of the magnetization governed by a Raman process and showing coherent spin

Received: November 6, 2019

Accepted: January 29, 2020

Published: January 29, 2020 
dynamics. We demonstrate that the Raman relaxation process is intrinsic to the molecule in this case and depends weakly on temperature $\propto T^{3}$ as compared to $T^{5}$ or $T^{9}$, postulated for Kramers ions. ${ }^{28}$ Moreover, this Raman relaxation depends on the dilution of $\mathbf{1}$ in the solid state matrix. Both factors are crucial from the point of view of designing high-efficiency lanthanide-based molecular qubits, which is clearly highlighted in our case study.

The magnetic properties of the complex cation [Gd(phendo) $\left.)_{4}\right]^{3+}$ were studied for the undiluted compound $\mathbf{1}$, for the methanolic solution (mass spectroscopy confirms the presence of this cation in methanol, as discussed in the SI), and for the three solid state diluted compounds prepared using its structural analog $\left[\mathrm{Y}(\text { phendo })_{4}\right]\left(\mathrm{NO}_{3}\right)_{3} \cdot x \mathrm{MeOH}$ (2) as a diamagnetic matrix $(2.73 \%, 0.47 \%$, and $0.17 \%$ concentrations of $\mathrm{Gd}^{\mathrm{III}}$ ions). Magnetically diluted samples were tested using pulse EPR spectroscopy to show long phase memory time $T_{\mathrm{m}}$ compared to other lanthanide-based qubits and fast Rabi oscillations at $20 \mathrm{~K}$.

Crystal Structure. $\left[\mathrm{Gd}(\text { phendo })_{4}\right]\left(\mathrm{NO}_{3}\right)_{3} \cdot x \mathrm{MeOH}(\mathbf{1})$ is obtained in the reaction of gadolinium(III) nitrate with 1,10 phenanthroline- $N, N^{\prime}$-dioxide (phendo) in methanol solution, which yields yellow plate-shaped crystals (see SI for details). Crystal structure of $\mathbf{1}$ was determined by single-crystal X-ray diffraction (Table S2 in SI). 1 crystallizes in the centrosymmetric tetragonal space group $I 4 / \mathrm{m}$. The $\mathrm{Gd} 1$ atom occupies a 4-fold rotation axis, and as a result, the asymmetric unit consists of only a quarter of the formula unit (Figure S1). Two of the nitrate anions also occupy a special position of high symmetry (a 4-fold rotation axis and a mirror plane), which leads to a severe disorder, while the third one is placed on a mirror plane and is only slightly disordered (the presence of nitrate anions was confirmed by IR spectroscopy; see SI for details). Due to the high symmetry, it was impossible to find methanol molecules in the structural model, even if disorder was taken into account, and therefore, the SQUEEZE procedure was applied with the resulting voids of $921 \AA^{3}$ and 275 electrons at $117 \mathrm{~K}\left(1125 \AA^{3}\right.$ and 251 electrons at RT). These values of electron density account for around 14 methanol molecules per unit cell, which leads to molecular volumes of 65.8 and $80.4 \AA^{3}$ per $\mathrm{MeOH}$ at $117 \mathrm{~K}$ and RT, respectively (consistent with the literature reports ${ }^{29}$ ). The relatively large solvent accessible voids are responsible for the crystal breaking following the solvent loss at room temperature.

$\mathrm{Gd}^{\mathrm{III}}$ in $\mathbf{1}$ is coordinated by four neutral phendo ligands through the oxygen atoms of the $\mathrm{N}$-oxide moieties. This constitutes the first example of a coordination complex involving this peculiar helical molecule, according to the CSD database search. Such a coordination environment of a $\mathrm{Gd}^{\mathrm{III}}$ ion results in a nearly perfect square antiprism geometry of its coordination sphere (Figure 1), as confirmed by SHAPE analysis (Table S3 in the SI). There are two relevant angles that define the distortion from an ideal square antiprism geometry: the twist angle $\varphi$ and the compression/elongation angle $\theta$. For the ideal geometry, these angles are $45^{\circ}$ and $54.7^{\circ}$, respectively. ${ }^{30}$ For $\left[\mathrm{Gd}(\text { phendo })_{4}\right]^{3+}, \varphi=54.0^{\circ}, \theta_{1}=61.4^{\circ}$, and $\theta_{2}=60.1^{\circ}$ at $117 \mathrm{~K}$ and $\varphi=54.5^{\circ}, \theta_{1}=61.2^{\circ}$ and $\theta_{2}=$ $60.3^{\circ}$ at room temperature (Figure 1 ).

The twist angle $\varphi$ is crucial to determine the point group at the metal site, which is important for the appropriate description of the crystal field and resulting splitting of the $m_{\mathrm{J}}$ states. As the twist angle $\varphi$ differs from $45^{\circ}$, the symmetry a)

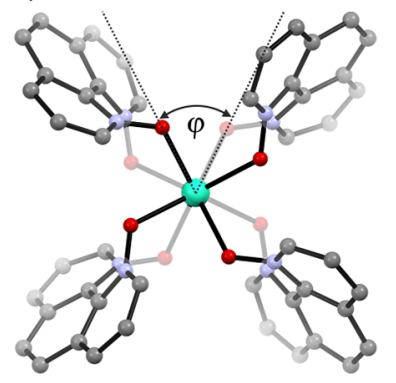

b)

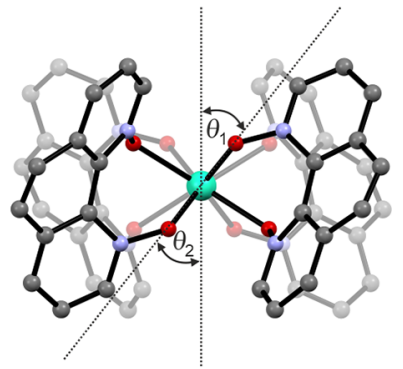

Figure 1. Structural diagram of the $\Delta$ stereoisomer of the $\left[\mathrm{Gd}(\text { phendo })_{4}\right]^{3+}$ propeller-like cation viewed along (a) and perpendicular (b) to its 4-fold symmetry axis: $\mathrm{Gd}$, green; $\mathrm{O}$, red; $\mathrm{N}$, blue; $\mathrm{C}$, gray; $\mathrm{H}$, omitted for clarity. Angles $\varphi$ and $\theta$ indicate the distortion of the coordination sphere from a perfect square antiprism.

of the gadolinium site changes from $D_{4 d}$ to $D_{4}$ with further inequivalence of the oxygen atoms $\mathrm{O} 1$ and $\mathrm{O} 2(\mathrm{Gd}-\mathrm{O} 1=$ $2.356(3), \mathrm{Gd}-\mathrm{O} 2=2.349(3)$; values at $117 \mathrm{~K})$, lowering the symmetry to $C_{4}$. Due to the axial chirality of the ligand, the obtained complexes are also chiral and show a four-bladed propeller geometry, with the unit cell containing their racemic mixture: two $\Delta$ and two $\Lambda$ enantiomers (Figure 1). The lanthanide centers are quite well separated from each other, with the shortest Gd ‥Gd distance equal to $10.45 \AA$ (along the $c$ crystallographic direction). $\left[\mathrm{Gd}(\text { phendo })_{4}\right]^{3+}$ cations are separated by a nitrate ion along the 4-fold symmetry axis. Along the $a$ direction, Gd $\cdots \mathrm{Gd}$ distances equal $a=15.34 \AA$. Each Gd $\mathrm{d}^{\mathrm{III}}$ ion has also four near neighbors in the $a b$-plane within $10.86 \AA$ (Figure S2).

Continuous Wave EPR and Ab Initio Calculations. In order to conduct detailed pulse EPR experiments and to study the spin dynamics of the complex cation $\left[\mathrm{Gd}(\text { phendo })_{4}\right]^{3+}$, solid state dilution samples of $\mathbf{1}$ in the isostructural $\left[\mathrm{Y}(\text { phendo })_{4}\right]\left(\mathrm{NO}_{3}\right)_{3} \cdot x \mathrm{MeOH}$ (2) were prepared with the intended $\mathrm{Gd}^{\mathrm{III}}$ molar fractions of $5 \%, 1 \%$, and $0.3 \%$ (synthesis and characterization are described in the SI). The identity and purity of the solid state dilution samples were confirmed by powder X-ray diffraction (Figure S4). The actual molar fractions of $\mathrm{Gd}^{\mathrm{III}}$ were determined with high accuracy by ICP-QMS analysis and are approximately 50\% smaller than the intended ones (see SI for details), resulting in the following chemical formulas: $\left[\mathrm{Gd}_{0.0273} \mathrm{Y}_{0.9727}(\text { phendo })_{4}\right]\left(\mathrm{NO}_{3}\right)_{3} \cdot x \mathrm{MeOH}$ (1Gd2.73\%), $\left[\mathrm{Gd}_{0.0047} \mathrm{Y}_{0.9953}(\text { phendo })_{4}\right]\left(\mathrm{NO}_{3}\right)_{3} \cdot x \mathrm{MeOH}$ (1Gd0.47\%), and $\left[\mathrm{Gd}_{0.0017} \mathrm{Y}_{0.9983}(\text { phendo })_{4}\right]\left(\mathrm{NO}_{3}\right)_{3} \cdot x \mathrm{MeOH}$ (1Gd0.17\%).

Continuous wave EPR spectroscopy was employed in order to estimate the crystal field splitting in the studied [Gd(phendo $\left.)_{4}\right]^{3+}$ cation. Fast relaxation causes strong broadening of the EPR signal for the undiluted sample 1 (Figure S11). Therefore, detailed studies in X-, K-, and Q-bands were performed for a powdered sample of $1 \mathrm{Gd0.47 \%}$ at $150 \mathrm{~K}$ (Figure S12, black lines). In principle, seven Stevens crystal field parameters are required to describe an EPR spectrum of the $C_{4}$-symmetry complex of $\operatorname{Ln}^{3+}: B_{2}{ }^{0}, B_{4}{ }^{0}, B_{4}{ }^{4}, B_{4}{ }^{-4}, B_{6}{ }^{0}, B_{6}{ }^{4}$, and $B_{6}{ }^{-4}$. However, in the case of 1 , the distortion from the ideal $D_{4}$ symmetry is negligible (the difference between the $\mathrm{Gd}-\mathrm{O} 1$ and $\mathrm{Gd}-\mathrm{O} 2$ bond lengths is extremely small, within $0.007(6) \AA$ ), so both $B_{4}{ }^{-4}$ and $B_{6}{ }^{-4}$ are expected to approach zero.

Still, the attempt to fit the EPR spectra using the remaining five parameters failed, because the splitting caused by the $B_{2}{ }^{0}$ 
parameter is smaller than the line width. Therefore, the final EasySpin $^{31}$ fits presented in Figure S12 (red lines, SI) were performed with only three crystal field parameters, $B_{2}{ }^{0}, B_{4}{ }^{0}$, and $B_{4}^{4}$, and the remaining variables describe the pseudo-Voigt line profile. The $g$ factor was determined from the Q-band spectrum and used as a fixed parameter in all other fits. This approach yielded the following best fit values: $g=1.991(1), B_{2}{ }^{0}$ $=8 \mathrm{MHz}\left(0.00027 \mathrm{~cm}^{-1}\right), B_{4}{ }^{0}=-0.9 \mathrm{MHz}\left(-0.00003 \mathrm{~cm}^{-1}\right)$, and $B_{4}{ }^{4}=16 \mathrm{MHz}\left(0.00053 \mathrm{~cm}^{-1}\right)$. These crystal field parameters are not sufficient to completely describe the crystal field of $\left.\left[\mathrm{Gd}^{\mathrm{III}} \text { (phendo) }\right)_{4}\right]^{3+}$ in $\mathbf{1}$, but allow the estimation of the total splitting of the ground $m_{\mathrm{J}}$ states, which was found to be $0.12 \mathrm{~cm}^{-1}$. This value is at least an order of magnitude smaller compared to the thermal energy, even at the lowest temperature achievable in the magnetic measurements $(1.8 \mathrm{~K}$ corresponds to $1.25 \mathrm{~cm}^{-1}$ ), hence, all $m_{\mathrm{J}}$ states are assumed to be equally populated, even at $1.8 \mathrm{~K}$.

Limiting the number of the CF parameters to three in the fitting of EPR data is fully supported by the results of the single-point $\mathrm{ab}$ initio calculations on the $\left[\mathrm{Gd}^{\mathrm{III}}(\text { phendo })_{4}\right]^{3+}$ cation performed using OpenMolcas ${ }^{33}$ (see Tables S4 and S5 in the SI for details). The calculated terms agree with the assumption that $B_{2}{ }^{0}, B_{4}{ }^{0}$, and $B_{4}{ }^{4}$ describe well the $\mathrm{CF}$ for $\left.\left[\mathrm{Gd}^{\mathrm{III}} \text { (phendo }\right)_{4}\right]^{3+}$, as the total weight of these three parameters on the crystal field splitting amounts to about 99\% (Table S4 in the SI). The calculated total splitting of the ground state is $0.48 \mathrm{~cm}^{-1}$ and confirms a very small magnetic anisotropy of the $\mathrm{Gd}^{\mathrm{III}}$ ion in $\mathbf{1}$.

Magnetic Properties. DC magnetic properties of $\mathbf{1}$ are typical for $\mathrm{Gd}^{\mathrm{III}}$ complexes and are discussed in the SI. Alternating current (AC) magnetic susceptibility measurements revealed an interesting dynamic behavior of 1 . In the $1.8-3.5 \mathrm{~K}$ temperature range under an applied DC field of 0.3 $\mathrm{T}$, the frequency dependence of the out-of phase $(\chi$ ") magnetic susceptibility shows two maxima in the $0.4-1000 \mathrm{~Hz}$ range (Figure S6c). This behavior is attributed to two relaxation processes, which were fitted using a generalized Debye model. However, it was impossible to obtain accurate $\tau$ values ( $\tau$, time of the spin-lattice relaxation of the magnetization) for the faster process, as the corresponding $\chi$ " maxima appear above $1000 \mathrm{~Hz}$. On the other hand, the slower relaxation process, which shows weak temperature dependence, is assumed to result from dipole-dipole interactions between $\mathrm{Gd}^{\mathrm{III}}$ ions, leading to cooperative relaxation of larger units. Therefore, in order to study this complicated relaxation behavior of the $\left[\mathrm{Gd}(\text { phendo })_{4}\right]^{3+}$ cation itself, solid state diluted samples 1Gd2.73\%, 1Gd0.47\%, and 1Gd0.17\% and the saturated solution of $\mathbf{1}$ in methanol (1 $\mathbf{s o l}$; see the mass spectrometry results in the SI confirming the presence of $\left.\left[\mathrm{Gd}^{\mathrm{III}} \text { (phendo }\right)_{4}\right]^{3+}$ cations in the $\mathrm{MeOH}$ solution) were also studied by AC magnetic susceptibility measurements. The aforementioned slower relaxation process is significantly reduced for $\mathbf{1 G d} 2.73 \%$ and 1 sol and vanishes completely for 1 Gd0.47\% and 1Gd0.17\%, as depicted in Figures S6-S10 in the SI. This confirms the initial assumption that this slower relaxation process is related to dipole-dipole interactions. The $\tau$ values extracted from the magnetic field $(H)$ dependencies of the AC magnetic susceptibility for the remaining faster process were fitted using the following equation: ${ }^{1}$

$$
\tau^{-1}(H)=A_{1} /\left(1+A_{2} H^{2}\right)+A_{3} H^{4}+A_{4}
$$

where the first part accounts for the quantum tunneling of magnetization (QTM), ${ }^{34}$ the second one is related to the direct relaxation process, ${ }^{35}$ and the last one stands for thermally dependent but field-independent processes (Orbach and Raman). ${ }^{36,37}$ The results obtained for all $\mathrm{Gd}^{\mathrm{III}}$ concentrations are presented in Figure 2 and summarized in Table 1.

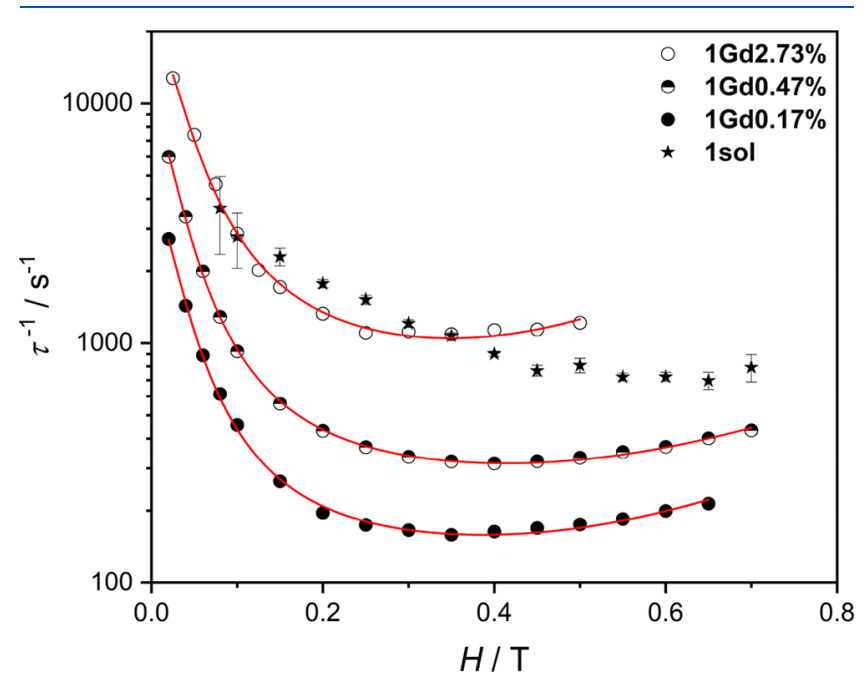

Figure 2. Magnetic field dependence of the relaxation time for diluted solid state samples of 1 (circles) and the solution 1sol (stars) obtained from the AC magnetic susceptibility measurements at $T=$ $1.8 \mathrm{~K}$. Solid lines represent the best fit to the model described in the text.

Both QTM and direct relaxation become less important with increasing dilution, as expected for the consequent weakening of the dipole-dipole interactions between the unpaired electron spins of the $\mathrm{Gd}^{\mathrm{III}}$ centers. In order to minimize contributions from these processes, temperature dependencies of $\chi^{\prime}$ and $\chi^{\prime \prime}$ were recorded under the optimal DC fields of 0.2 $\mathrm{T}$ for $1 \mathrm{Gd} 2.73 \%$ and $0.35 \mathrm{~T}$ for $1 \mathrm{Gd} 0.47 \%, 1 \mathrm{Gd} 0.17 \%$, and 1sol (Figures S7-S10). The obtained magnetic relaxation times $\tau$ at different temperatures $T$ were fitted using the following equation: ${ }^{38}$

$$
\tau^{-1}(T)=C_{0}+C_{1} T^{n}+C_{2} T^{m}
$$

where $C_{0}$ corresponds to QTM, $C_{1}$ and $n$ describe a Raman process, and $C_{2}$ and $m$ correspond to the direct process. In principle, a direct process can be described with $m=1$ for a typical spin-lattice relaxation and $m=2$ for a spin-phonon bottleneck process. However, we assume that for diluted powder samples relaxation to the phonon bath is sufficiently fast and does not limit the energy transfer from a few $\mathrm{Gd}^{\mathrm{III}}$ ions in the lattice of the solid state diluted compounds. This assumption is in accord with the observation of a similar slow magnetic relaxation in a frozen solution of $\left[\mathrm{Gd}^{\mathrm{III}}(\text { phendo })_{4}\right]$ $\left(\mathrm{NO}_{3}\right)_{3} \cdot x \mathrm{MeOH} 1 \mathrm{sol}\left(c=3(1) \times 10^{-3} \mathrm{~mol} / \mathrm{dm}^{3}\right.$; note that, in the case of $1 \mathrm{Gd} 0.17 \%$, the molar concentration equals $2.2 \times$ $10^{-3} \mathrm{~mol} / \mathrm{dm}^{3}$ ). The control of the relaxation by spin-lattice processes rather than a spin-phonon bath is unusual for a $\mathrm{Gd}^{\mathrm{III}}$ compound, ${ }^{20,22,26}$ but not unprecedented among magnetically isotropic systems. ${ }^{39,40}$ Therefore, the $\tau^{-1}(T)$ was fitted assuming that $m=1$ and $C_{0}$ and $C_{2}$ are fixed based on the results of the $\tau^{-1}(H)$ (eq 1 ), while $C_{1}$ and $n$ are the only free parameters (except for 1sol, where $C_{0}$ was treated as a free parameter, as it was impossible to determine it from $\tau^{-1}(H)$ dependence). The Orbach process does not influence the 
Table 1. Fit Parameters Obtained from AC and EPR Measurements for 1Gd2.73\%, 1Gd0.47\%, 1Gd0.17\%, and 1sol

\begin{tabular}{|c|c|c|c|c|}
\hline & $1 G d 2.73 \%$ & 1Gd0.47\% & 1Gd0.17\% & 1 sol \\
\hline$A_{1}\left(\mathrm{~s}^{-1}\right)$ & $1.8(2) \times 10^{4}$ & $8.5(3) \times 10^{3}$ & $3.7(2) \times 10^{3}$ & $a$ \\
\hline$A_{2}\left(\mathrm{~T}^{-2}\right)$ & $8(1) \times 10^{2}$ & $1.13(5) \times 10^{3}$ & $1.08(9) \times 10^{3}$ & $a$ \\
\hline$A_{3}\left(\mathrm{~T}^{-4}\right)$ & $6.5(13) \times 10^{3}$ & $7.4(3) \times 10^{2}$ & $50(4)$ & $a$ \\
\hline$A_{4}\left(\mathrm{~s}^{-1}\right)$ & $7.6(5) \times 10^{2}$ & $2.50(4) \times 10^{2}$ & $1.24(3) \times 10^{2}$ & $a$ \\
\hline$C_{0}\left(\mathrm{~s}^{-1}\right)$ & 550 (fixed) & 61 (fixed) & 28 (fixed) & $3.5(2) \times 10^{2}$ \\
\hline$C_{1}\left(\mathrm{~s}^{-1} \mathrm{~K}^{-n}\right)$ & $1.2(2) \times 10^{2}$ & $56(1)$ & $26(1)$ & $86(5)$ \\
\hline$n$ & $2.74(14)$ & $2.67(2)$ & $2.98(3)$ & $2.91(4)$ \\
\hline$C_{2}\left(\mathrm{~s}^{-1} \mathrm{~K}^{-1}\right)$ & 5.8 (fixed) & 6.2 (fixed) & 0.42 (fixed) & $0(\text { fixed })^{b}$ \\
\hline$C_{1}^{\prime}\left(\mathrm{s}^{-1} \mathrm{~K}^{-n^{\prime}}\right)^{c}$ & & $6(3)$ & $16(7)$ & \\
\hline$n^{\prime}$ & & $2.9(1)$ & $2.8(1)$ & \\
\hline$C_{2}^{\prime}\left(\mathrm{s}^{-1} \mathrm{~K}^{-1}\right)$ & & $1.1(1) \times 10^{3}$ & $5.8(1) \times 10^{2}$ & \\
\hline
\end{tabular}

${ }^{a}$ Wide distribution of relaxation times in the field dependence of the AC signal for 1 sol ( $\alpha$ approaching 0.36) does not enable a reliable fit to eq 1 . ${ }^{b}$ As no significant decrease of relaxation time is observed in the field dependence of the AC signal up to $0.7 \mathrm{~T}$ (Figure 2 ), the direct process is assumed to be negligible under $H=0.35 \mathrm{~T}$. ${ }^{c}$ Primed parameters are obtained from EPR data.

magnetization dynamics due to the total splitting of the $m_{\mathrm{J}}$ multiplet lower than $0.3 \mathrm{~K}$, leading to an almost equal population of all $m_{\mathrm{J}}$ states above $1.8 \mathrm{~K}$. The fitting results are presented in Table 1 and Figure 3. The obtained $n$ values

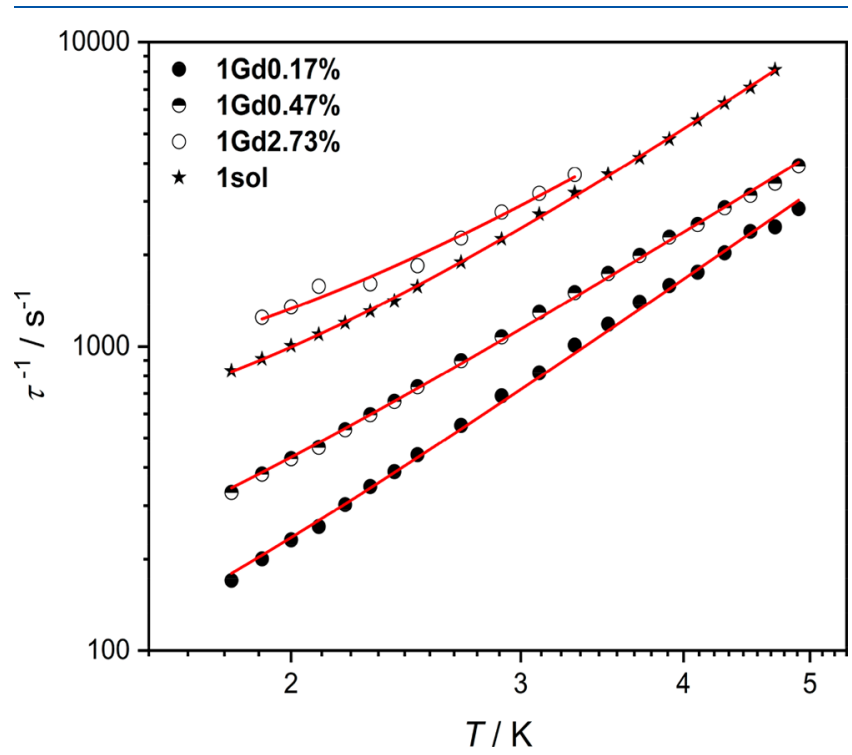

Figure 3. Thermal dependencies of $\tau^{-1}$ for magnetically diluted samples of 1 and 1 sol $\left(H_{\mathrm{DC}}=0.20 \mathrm{~T}\right.$ for $1 \mathrm{Gd} 2.73 \%$ and $H_{\mathrm{DC}}=0.35$

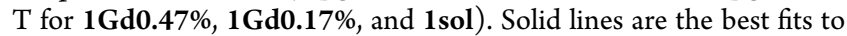
the eq 2 (see details in text and Table 1).

approach $n=3$ for both solid state dilutions as well as for 1sol, suggesting a two-phonon Raman-like relaxation process involving both optical and acoustic phonons. ${ }^{41,42}$ The similar thermal dependence of $\tau$ for solid state diluted samples and a $\mathrm{MeOH}$ solution confirms that the studied slow magnetic relaxation is an intrinsic "molecular" property of the [Gd(phendo) $\left.)_{4}\right]^{3+}$ cation and not the result of slow phonon exchange with the thermal bath (phonon bottleneck effect).

Pulse EPR. In order to further confirm that the magnetization dynamics of $\mathbf{1}$ is controlled by the Raman-like process $\left(\tau^{-1}=C T^{3}\right)$ and not phonon-bottleneck $\left(\tau^{-1}=C T^{2}\right)$, we performed pulse EPR experiments (X-band; $9.24 \mathrm{GHz}$ ). All diluted samples show intense echo-detected signal which remains intense up to $30 \mathrm{~K}$ for $\mathbf{1 G d 0 . 4 7 \%}$ (Figure 4) and 1Gd0.17\% (Figure S13) and vanishes fast for 1Gd2.73\%, (Figure S14). In order to elucidate the spin-lattice relaxation

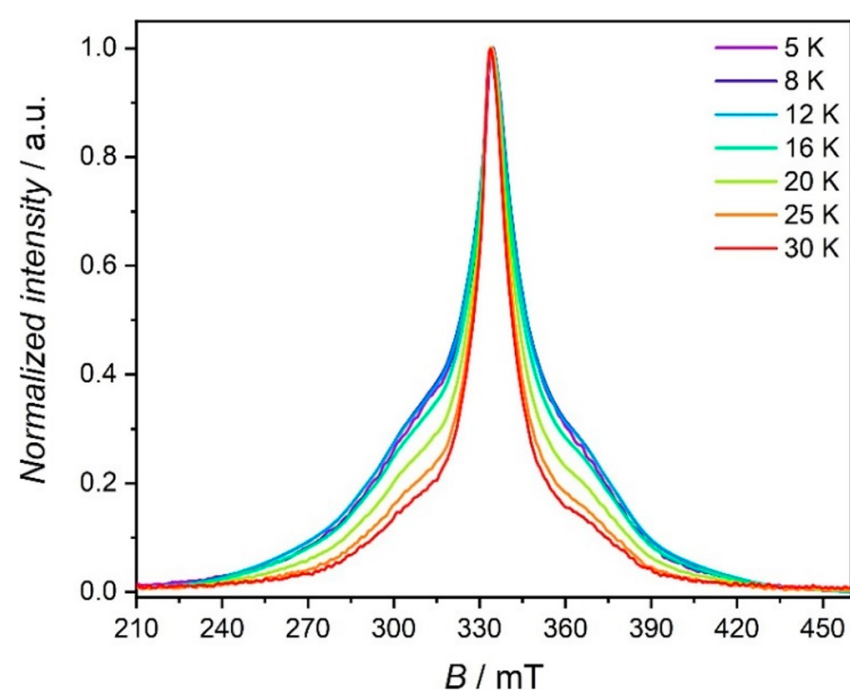

Figure 4. Field-swept echo-detected EPR spectra (9.24 GHz; $\pi / 2$ pulse of $128 \mathrm{~ns}$ ) of $\mathbf{1 G d 0 . 4 7 \%}$.

times, inversion recovery sequence traces at $335 \mathrm{mT}$ were fitted to the biexponential model to afford two time constants (Figures S15-S17). The longer one is assumed to be spinlattice relaxation time, $T_{1}$, and the shorter one is ascribed to the spectral diffusion, $T_{1, s^{*}}{ }^{4}$ This assumption is in accordance with the literature ${ }^{44-49}$ and is additionally confirmed by the similarity of these $T_{1}$ values with $\tau$ obtained from AC magnetic susceptibility for the solid state diluted compounds. Note that both $\tau$ and $T_{1}$ represent the same physical quantity-the time of spin-lattice relaxation of magnetization. $T_{1, s}$, on the other hand, is found to be $5-10 \times$ shorter than $T_{1}$ (Tables S6-S8). The obtained $T_{1}$ values were fitted to eq 2 , with the $C_{0}$ derived from the AC magnetic susceptibility measurements and $m=1$. Results are presented in Figure S18 and Table $1\left(C_{1}{ }^{\prime}\right.$ and $\left.n^{\prime}\right)$. Both AC and EPR measurements yield similar $n$ values, while $C_{2}(\mathrm{AC})$ and $C_{2}{ }^{\prime}(\mathrm{EPR})$ differ significantly because pulse EPR experiment probes the relaxation times of a discrete transition centered at $335 \mathrm{mT}$ and the magnetic measurements determine the averaged relaxation time for all $m_{\mathrm{J}}$ states. Moreover, the $T_{1}$ values obtained from EPR below $8 \mathrm{~K}$ are affected by the spectral diffusion which is much faster than spin-phonon relaxation at low temperatures. Nonetheless, both methods show that $n=3$ Raman-like process is the fastest 
relaxation pathway for $\left[\mathrm{Gd}^{\mathrm{III}} \text { (phendo) }{ }_{4}\right]^{3+}$ and orchestrates the spin-lattice relaxation in both $1 \mathrm{Gd} 0.47 \%$ and $1 \mathrm{Gd} 0.17 \%$.

$\left.\left[\mathrm{Gd}^{\mathrm{III}} \text { (phendo }\right)_{4}\right]^{3+}$ is characterized by a nuclear spin-free coordination sphere, which limits the decoherence and is promising in terms of achieving long memory phase times. As the $T_{1}$ is sufficiently long not to limit the quantum coherence time $\left(T_{\mathrm{m}}\right)$, we tested the temperature-dependence of $T_{\mathrm{m}}$ for the diluted gadolinium samples by recording the time decay of the Hahn echo (Figures S19 and S20 and Tables S9-S11). For 1Gd2.73\%, $T_{\mathrm{m}}$ was found to be relatively short (around $150 \mathrm{~ns}$ in 5-10 K range), while for both $1 \mathrm{Gd} 0.47 \%$ and $1 \mathrm{Gd0.17 \%}$, the $T_{\mathrm{m}}$ values exceed $2.5 \mu \mathrm{s}$ at $5 \mathrm{~K}$ (Figure 5 ). This value is

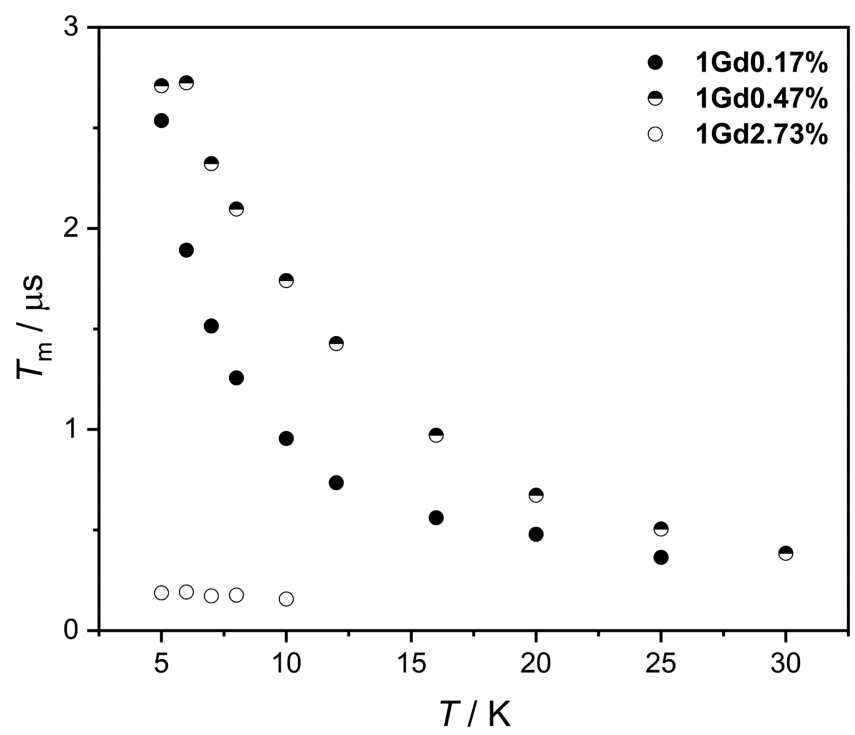

Figure 5. Phase memory time $T_{\mathrm{m}}$ temperature-dependence for magnetically diluted $\mathbf{1}$.

surpassed by the atomic clock transitions in $\left[\mathrm{Ho}\left(\mathrm{W}_{5} \mathrm{O}_{18}\right)_{2}\right]^{9-50}$ or gadolinium-doped $\mathrm{CaWO}_{4}$ crystal $^{51}$ and far from those obtained for record-breaking transition metal based qubits, ${ }^{44,52,53}$ but remains higher than reported for other lanthanide-based molecular qubits ${ }^{17,21,46,54}$ and is comparable with some obtained for transition metals such as $2.7 \mu \mathrm{s}$ for $\mathrm{VO}(\mathrm{dpm})_{2}{ }^{42}$ and $2.4 \mu \mathrm{s}$ for $\left(\mathrm{Ph}_{4} \mathrm{P}\right)_{3}\left[\mathrm{Fe}(\mathrm{CN})_{6}\right]^{55}$ under identical conditions. Surprisingly, 1Gd0.47\% shows systematically longer $T_{\mathrm{m}}$ than the more diluted $1 \mathrm{Gd0.17 \%}$. Taking into account blocking of QTM under applied magnetic field and small contribution of direct relaxation process (Figure S22), the Raman process becomes a dominant magnetization relaxation mechanism in magnetically diluted $\mathbf{1}$. Therefore, optimization of this process becomes crucial for control of $T_{1}$ in similar assemblies, which is known to be a limiting factor for $T_{\mathrm{m}}{ }^{56}$ For the studied compounds Raman process coefficient $C_{1}$ is decreasing with gadolinium concentration in yttrium matrix, ranging from $2.73 \%$ to $0.17 \%$ (Table 1 ). This observation can be explained on the basis of diminishing number of gadolinium(III) spins interacting with phonons, while the latter should remain almost unchanged upon proceeding from $99.83 \%$ to $97.27 \%$ yttrium(III) in the crystal. However, the observed slowing of relaxation does not seem to depend linearly on concentration. Particularly, when the Raman process efficiency $\left(\Gamma_{\text {Raman }}=C_{1} T^{3}\right)$ is divided by gadolinium(III) concentration to account for the probability of the relaxation of a single gadolinium(III) ion, the resulting efficiency of a single center relaxation seems to be increasing with dilution (Figure S23). Possibly two-phonon relaxation mechanism may be favored by a diminishing of the direct process in $1 \mathrm{Gd} 0.17 \%$, increasing efficiency of spin interaction with high-energy phonons. However, this behavior, which may be contributing to the unexpected shortening of the $T_{\mathrm{m}}$ in $1 \mathrm{Gd0.17 \%}$, as compared to $1 \mathrm{Gd} 0.47 \%$, needs further studies for a wider series of concentrations that will be conducted in future.

The possibility to coherently manipulate the spin states in 1Gd0.47\% and $1 \mathrm{Gd0.17 \%}$ was additionally probed by transient nutation experiments at $20 \mathrm{~K}$. The resulting Rabi oscillations for $1 \mathrm{Gd0.47 \%}$ and $\mathbf{1 G d 0 . 1 7 \%}$ are presented in Figures 6 and S24, respectively. Their frequencies were derived

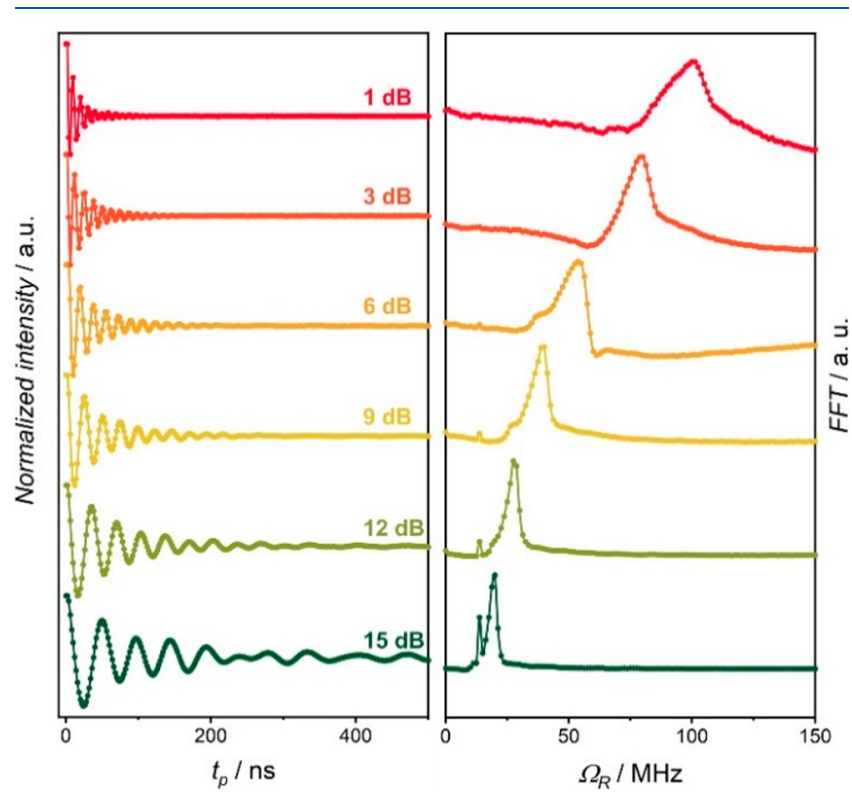

Figure 6. Normalized echo intensity showing the result of nutation experiments on $1 \mathrm{Gd} 0.47 \%$ at $20 \mathrm{~K}$ and different microwave powers (a) and their corresponding Fourier transforms (b).

from the fast Fourier transforms (Figure 6, right panel) and are linearly dependent on $B_{1}$, unlike the small feature at $15 \mathrm{MHz}$ which is power-independent and coincides with the Larmor frequency of hydrogen nucleus (Figures S25 and S26). Very fast Rabi oscillations $\left(\Omega_{\mathrm{R}} \approx 100 \mathrm{MHz}\right)$ are observed at the highest microwave power, which in principle could afford multiple Rabi oscillations given $N \approx \Omega_{\mathrm{R}} T_{2}>50$ at $20 \mathrm{~K}$. However, much faster damping limits their number which is most likely caused by the inhomogeneity in the nutation frequency. ${ }^{57}$ An enhanced decoherence demonstrated by shorter $T_{\mathrm{m}}$ times for $\mathbf{1 G d 0 . 1 7 \%}$ is also observed in the nutation experiments, as number of Rabi oscillations that can

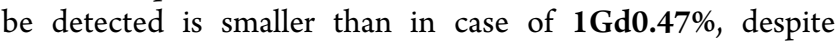
higher dilution of a former (Figure S24).

We have extensively investigated the slow relaxation of the magnetization of the propeller-like gadolinium(III)-based single ion magnet in its $\left.\left[\mathrm{Gd}^{\mathrm{III}} \text { (phendo }\right)_{4}\right]\left(\mathrm{NO}_{3}\right)_{3} \cdot x \mathrm{MeOH}$ salt and its solid state dilutions in the diamagnetic $\left.\left[\mathrm{Y}^{\mathrm{III}} \text { (phendo }\right)_{4}\right]\left(\mathrm{NO}_{3}\right)_{3} \cdot x \mathrm{MeOH}$ matrix. The relaxation of the diluted compounds is driven dominantly by the Raman-like process with the relaxation time $\tau \propto T^{-3}$, which is unusual for Kramers ions. The observation of the slow relaxation of magnetization for both the frozen methanol solution of 
$\left.\left[\mathrm{Gd}^{\mathrm{III}} \text { (phendo }\right)_{4}\right]\left(\mathrm{NO}_{3}\right)_{3} \cdot x \mathrm{MeOH}$, as well as the solid state dilution compounds excludes the phonon bottleneck effect as its source and highlights the importance of the Raman relaxation process in designing lanthanide-based qubits. This is supported by the observation of the increase of the Raman relaxation efficiency at the highest Gd:Y dilution. Long $T_{1}$ and $T_{\mathrm{m}}$ times enabled the observation of Rabi oscillations at $20 \mathrm{~K}$, confirming the coherent spin state control in $\left.\left[\mathrm{Gd}^{\mathrm{III}} \text { (phendo }\right)_{4}\right]^{3+}$. Further studies concerning the surprisingly longer $T_{\mathrm{m}}$ for $\mathbf{1 G d 0 . 4 7 \%}$ as compared to $\mathbf{1 G d 0 . 1 7 \%}$ and attempts to obtain pure chiral salts of $\left.\left[\mathrm{Gd}^{\mathrm{III}} \text { (phendo) }\right)_{4}\right]^{3+}$ with enantiopure anions or through the exploitation of chiralinduced spin selectivity effect (CISS) ${ }^{58}$ are in progress.

\section{EXPERIMENTAL METHODS}

All experimental details can be found in the Supporting Information. This includes the following: (1) Synthesis details; (2) Elemental analysis; (3) Single-crystal X-ray diffraction; (4) Shape calculations; (5) Powder X-ray diffraction; (6) Magnetic measurements; (7) Ab initio calculations; (8) EPR spectroscopy; (9) Inductively coupled plasma mass spectrometry and emission spectroscopy; (10) Mass spectrometry; (11) Infrared spectroscopy.

\section{ASSOCIATED CONTENT}

\section{SI Supporting Information}

The Supporting Information is available free of charge at https://pubs.acs.org/doi/10.1021/acs.jpclett.9b03275.

Synthesis of the reported compounds, elemental analyses, single crystal, and powder X-ray diffraction, ICP-MS analyses, mass spectrometry, IR and EPR spectroscopy, additional magnetic plots, and the information about the cif files for $\mathbf{1}$ and $\mathbf{2}$ (available free of charge from The Cambridge Crystallographic Data Centre via www.ccdc.cam.ec.uk/data_request/cif under the CCDC 1944789-1944792 deposition numbers) (PDF)

Molcas input file (TXT)

\section{AUTHOR INFORMATION}

\section{Corresponding Author}

Dawid Pinkowicz - Faculty of Chemistry, Jagiellonian University, 30-387 Kraków, Poland; 이이이.org/0000-00029958-3116; Email: dawid.pinkowicz@uj.edu.pl

\section{Authors}

Gabriela Handzlik - Faculty of Chemistry, Jagiellonian University, 30-387 Kraków, Poland

Michał Magott - Faculty of Chemistry, Jagiellonian University, 30-387 Kraków, Poland; (1) orcid.org/0000-0002-4566-2636

Mirosław Arczyński - Faculty of Chemistry, Jagiellonian University, 30-387 Kraków, Poland

Alena M. Sheveleva - School of Chemistry and Photon Science Institute, The University of Manchester, Manchester M13 9PL, United Kingdom

Floriana Tuna - School of Chemistry and Photon Science Institute, The University of Manchester, Manchester M13 9PL, United Kingdom

Marcin Sarewicz - Department of Molecular Biophysics, Faculty of Biochemistry, Biophysics and Biotechnology, Jagiellonian University, 30-387 Krakow, Poland
Artur Osyczka - Department of Molecular Biophysics, Faculty of Biochemistry, Biophysics and Biotechnology, Jagiellonian University, 30-387 Kraków, Poland; 이이.org/0000-00021637-2365

Michał Rams - Marian Smoluchowski Institute of Physics, Jagiellonian University, 30-348 Kraków, Poland

Veacheslav Vieru - Theory and Nanomaterials Group, Katholieke Universiteit Leuven, 3001 Leuven, Belgium; (1) orcid.org/0000-0001-6375-4537

Liviu F. Chibotaru - Theory and Nanomaterials Group, Katholieke Universiteit Leuven, 3001 Leuven, Belgium

Complete contact information is available at: https://pubs.acs.org/10.1021/acs.jpclett.9b03275

\section{Author Contributions}

${ }^{\dagger}$ These authors contributed equally to this work.

\section{Notes}

The authors declare no competing financial interest.

\section{ACKNOWLEDGMENTS}

Financial support from the Polish Ministry of Science and Higher Education within the Diamond Grant (0191/DIA/ 2017/46), the Polish National Center within the Sonata Bis Project (2016/22/E/ST5/00055), and the MolSpin COST action CA15128 is gratefully acknowledged. We thank the EPSRC UK National EPR Facility at the University of Manchester for EPR measurements, Leverhulme Trust UK for a research fellowship to F.T. (RF-2018-545\4), and the Royal Society for a Newton International Fellowship to A.S. $(\mathrm{NIF} \backslash \mathrm{R} 1 \backslash 180441)$. V.V. acknowledges the postdoctoral fellowships from the Fonds Wetenschappelijk OnderzoekVlaanderen (FWO, Flemish Science Foundation). This research was also supported in part by PL-Grid Infrastructure.

\section{REFERENCES}

(1) Gatteschi, D.; Sessoli, R.; Villain, J. Molecular Nanomagnets; Oxford University Press, 2006.

(2) Woodruff, D. N.; Winpenny, R. E. P.; Layfield, R. A. Lanthanide Single-Molecule Magnets. Chem. Rev. 2013, 113, 5110-5148.

(3) Goodwin, C. A. P.; Ortu, F.; Reta, D.; Chilton, N. F.; Mills, D. P. Molecular Magnetic Hysteresis at $60 \mathrm{~K}$ in Dysprosocenium. Nature 2017, 548, 439-442.

(4) Guo, F.-S.; Day, B. M.; Chen, Y.-C.; Tong, M.-L.; Mansikkamäki, A.; Layfield, R. A. A Dysprosium Metallocene Single-Molecule Magnet Functioning at the Axial Limit. Angew. Chem., Int. Ed. 2017, 56, 11445-11449.

(5) Guo, F.-S.; Day, B. M.; Chen, Y.-C.; Tong, M.-L.; Mansikkamäki, A.; Layfield, R. A. Magnetic Hysteresis $\mathrm{Up}$ to $80 \mathrm{~K}$ in a Dysprosium Metallocene Single-Molecule Magnet. Science 2018, 362, 1400-1403.

(6) Ungur, L.; Chibotaru, L. F. Strategies toward High-Temperature Lanthanide-Based Single-Molecule Magnets. Inorg. Chem. 2016, 55, 10043-10056.

(7) Abbasi, P.; Quinn, K.; Alexandropoulos, D. I.; Damjanović, M.; Wernsdorfer, W.; Escuer, A.; Mayans, J.; Pilkington, M.; Stamatatos, T. C. Transition Metal Single-Molecule Magnets: A $\left\{\mathrm{Mn}_{31}\right\}$ Nanosized Cluster with a Large Energy Barrier of $\sim 60 \mathrm{~K}$ and Magnetic Hysteresis at $~ 5$ K. J. Am. Chem. Soc. 2017, 139, 1564415647.

(8) Rinehart, J. D.; Long, J. R. Exploiting Single-Ion Anisotropy in the Design of f-element Single-Molecule Magnets. Chem. Sci. 2011, 2, 2078-2085.

(9) Scott, P. L.; Jeffries, C. D. Spin-Lattice Relaxation in Some RareEarth Salts at Helium Temperatures; Observation of the Phonon Bottleneck. Phys. Rev. 1962, 127, 32-51. 
(10) Escalera-Moreno, L.; Baldoví, J. J.; Gaita-Ariño, A.; Coronado, E. Spin states, vibrations and spin relaxation in molecular nanomagnets and spin qubits: a critical perspective. Chem. Sci. 2018, 9, 3265-3275.

(11) Pedersen, K. S.; Dreiser, J.; Weihe, H.; Sibille, R.; Johannesen, H. V.; Sørensen, M. A.; Nielsen, B. E.; Sigrist, M.; Mutka, H.; Rols, S.; Bendix, J.; Piligkos, S. Design of Single-Molecule Magnets: Insufficiency of the Anisotropy Barrier as the Sole Criterion. Inorg. Chem. 2015, 54, 7600-7606.

(12) Pointillart, F.; Bernot, K.; Golhen, S.; Le Guennic, B.; Guizouarn, T.; Ouahab, L.; Cador, O. Magnetic Memory in an Isotopically Enriched and Magnetically Isolated Mononuclear Dysprosium Complex. Angew. Chem., Int. Ed. 2015, 54, 1504-1507. (13) Giansiracusa, M. J.; Kostopoulos, A. K.; Collison, D.; Winpenny, R. E. P.; Chilton, N. F. Correlating Blocking Temperatures with Relaxation Mechanism in Monometallic Single-Molecule Magnets with High Energy Barriers $\left(U_{\text {eff }}>600 \mathrm{~K}\right)$. Chem. Commun. 2019, 55, 7025-7028.

(14) Liu, J.-L.; Chen, Y.-C.; Tong, M.-L. Symmetry Strategies for High Performance Lanthanide-based Single-Molecule Magnets. Chem. Soc. Rev. 2018, 47, 2431-2453.

(15) Gaita-Ariño, A.; Luis, F.; Hill, S.; Coronado, E. Molecular Spins for Quantum Computation. Nat. Chem. 2019, 11, 301-309.

(16) Atzori, M.; Sessoli, R. The Second Quantum Revolution: Role and Challenges of Molecular Chemistry. J. Am. Chem. Soc. 2019, 141, 11339-11352.

(17) Jenkins, M. D.; Duan, Y.; Diosdado, B.; Garcia-Ripoll, J. J.; Gaita-Arino, A.; Gimenez-Saiz, C.; Alonso, P. J.; Coronado, E.; Luis, F. Coherent Manipulation of Three-Qubit States in a Molecular Single-Ion Magnet. Phys. Rev. B: Condens. Matter Mater. Phys. 2017, 95, 064423.

(18) Hussain, R.; Allodi, G.; Chiesa, A.; Garlatti, E.; Mitcov, D.; Konstantatos, A.; Pedersen, K. S.; De Renzi, R.; Piligkos, S.; Carretta, S. Coherent Manipulation of a Molecular Ln-Based Nuclear Qudit Coupled to an Electron Qubit. J. Am. Chem. Soc. 2018, 140, 98149818.

(19) Ding, Y.-S.; Deng, Y.-F.; Zheng, Y.-Z. The Rise of Single-Ion Magnets as Spin Qubits. Magnetochemistry 2016, 2, 40.

(20) Orendac, M.; Sedlakova, L.; Cizmar, E.; Orendacova, A.; Feher, A.; Zvyagin, S. A.; Wosnitza, J.; Zhu, W. H.; Wang, Z. M.; Gao, S. Spin Relaxation and Resonant Phonon Trapping in $\left[\mathrm{Gd}_{2}(\text { fum })_{3}\left(\mathrm{H}_{2} \mathrm{O}\right)_{4}\right] \cdot 3 \mathrm{H}_{2} \mathrm{O}$. Phys. Rev. B: Condens. Matter Mater. Phys. 2010, 81, 214410.

(21) Martínez-Pérez, M. J.; Cardona-Serra, S.; Schlegel, C.; Moro, F.; Alonso, P. J.; Prima-García, H.; Clemente-Juan, J. M.; Evangelisti, M.; Gaita-Ariño, A.; Sesé, J.; van Slageren, J.; Coronado, E.; Luis, F. Gd-Based Single-Ion Magnets with Tunable Magnetic Anisotropy: Molecular Design of Spin Qubits. Phys. Rev. Lett. 2012, 108, 247213. (22) Arauzo, A.; Lazarescu, A.; Shova, S.; Bartolomé, E.; Cases, R.; Luzón, J.; Bartolomé, J.; Turta, C. Structural and Magnetic Properties of Some Lanthanide ( $\mathrm{Ln}=\mathrm{Eu}(\mathrm{III}), \mathrm{Gd}(\mathrm{III})$ and $\mathrm{Nd}(\mathrm{III})$ ) Cyanoacetate Polymers: Field-Induced Slow Magnetic Relaxation in the Gd and Nd Substitutions. Dalton Trans 2014, 43, 12342-12356. (23) Holmberg, R. J.; Ho, L. T. A.; Ungur, L.; Korobkov, I.; Chibotaru, L. F.; Murugesu, M. Observation of Unusual SlowRelaxation of the Magnetization in a Gd-EDTA Chelate. Dalton Trans 2015, 44, 20321-20325.

(24) Yoshida, T.; Cosquer, G.; Izuogu, D. C.; Ohtsu, H.; Kawano, M.; Lan, Y.; Wernsdorfer, W.; Nojiri, H.; Breedlove, B. K.; Yamashita, M. Field-Induced Slow Magnetic Relaxation of $\mathrm{Gd}^{\mathrm{III}}$ Complex with a Pt-Gd Heterometallic Bond. Chem. - Eur. J. 2017, 23, 4551-4556.

(25) Izuogu, D. C.; Yoshida, T.; Zhang, H.; Cosquer, G.; Katoh, K.; Ogata, S.; Hasegawa, M.; Nojiri, H.; Damjanović, M.; Wernsdorfer, W.; Uruga, T.; Ina, T.; Breedlove, B. K.; Yamashita, M. Slow Magnetic Relaxation in a Palladium-Gadolinium Complex Induced by Electron Density Donation from the Palladium Ion. Chem. - Eur. J. 2018, 24, 9285-9294.
(26) Girginova, P. I.; Pereira, L. C. J.; Coutinho, J. T.; Santos, I. C.; Almeida, M. Slow Magnetic Relaxation in Lanthanide Ladder Type Coordination Polymers. Dalton Trans 2014, 43, 1897-1905.

(27) Rozen, S.; Dayan, S. At Last, 1,10-Phenanthroline-N,N'dioxide, A New Type of Helicene, has been Synthesized using HOF. $\mathrm{CH}_{3} \mathrm{CN}$. Angew. Chem., Int. Ed. 1999, 38, 3471-3473.

(28) Carlin, R. L. Magnetochemistry; Springer: Berlin, Heidelberg, 1986.

(29) Leben, L.; Näther, C.; Herges, R. Crystal Structure of (methanol- $\kappa O)[5,10,15,20$-tetrakis (2-aminophenyl)porphyrinato$\left.\kappa^{4} \mathrm{~N}\right]$ zinc(II)-chloroform-methanol (1/1/1). Acta Cryst. E 2018, 74, $1285-1289$

(30) Sørensen, M. A.; Weihe, H.; Vinum, M. G.; Mortensen, J. S.; Doerrer, L. H.; Bendix, J. Imposing High-Symmetry and Tunable Geometry on Lanthanide Centres with Chelating $\mathrm{Pt}$ and $\mathrm{Pd}$ Metalloligands. Chem. Sci. 2017, 8, 3566-3575.

(31) Stoll, S.; Schweiger, A. EasySpin, a Comprehensive Software Package for Spectral Simulation and Analysis in EPR. J. Magn. Reson. 2006, 178, 42-55.

(32) Chilton, N. F.; Anderson, R. P.; Turner, L. D.; Soncini, A.; Murray, K. S. PHI: A Powerful New Program for the Analysis of Anisotropic Monomeric and Exchange-Coupled Polynuclear $d$ - and $f$ block Complexes. J. Comput. Chem. 2013, 34, 1164-1175.

(33) Aquilante, F.; Autschbach, J.; Carlson, R. K.; Chibotaru, L. F.; Delcey, M. G.; De Vico, L.; Fdez Galván, I.; Ferré, N.; Frutos, L. M.; Gagliardi, L.; Garavelli, M.; Giussani, A.; Hoyer, C. E.; Li Manni, G.; Lischka, H.; Ma, D.; Malmqvist, P. Å.; Müller, T.; Nenov, A.; Olivucci, M.; Pedersen, T. B.; Peng, D.; Plasser, F.; Pritchard, B.; Reiher, M.; Rivalta, I.; Schapiro, I.; Segarra-Martí, J.; Stenrup, M.; Truhlar, D. G.; Ungur, L.; Valentini, A.; Vancoillie, S.; Veryazov, V.; Vysotskiy, V. P.; Weingart, O.; Zapata, F.; Lindh, R. MOLCAS 8: New Capabilities for Multiconfigurational Quantum Chemical Calculations Across the Periodic Table. J. Comput. Chem. 2016, 37, 506-541.

(34) Van Vleck, J. H. Paramagnetic Relaxation Times for Titanium and Chrome Alum. Phys. Rev. 1940, 57, 426-447.

(35) Soeteman, J.; Bevaart, L.; Van Duyneveldt, A. J. The Direct and Raman Spin-Lattice Relaxation Process in $\mathrm{YbCl}_{3} \cdot 6 \mathrm{H}_{2} \mathrm{O}$. Physica 1974, $74,126-134$.

(36) Orbach, R. On the Theory of Spin-Lattice Relaxation in Paramagnetic Salts. Proc. Phys. Soc., London 1961, 77, 821-826.

(37) Orbach, R. Spin-Lattice Relaxation in Rare-Earth Salts: Field Dependence of the Two-Phonon Process. Proc. R. Soc. London 1961, 264, 485-495.

(38) Eaton, S. S.; Eaton, G. R. Chapter 9: Relaxation Mechanisms. In EPR Spectroscopy: Fundamentals and Methods; Goldfarb, D., Stoll, S., Eds.; Wiley, 2018; pp 175-192.

(39) Boča, R.; Rajnák, C.; Titiš, J.; Valigura, D. Field Supported Slow Magnetic Relaxation in a Mononuclear $\mathrm{Cu}(\mathrm{II})$ Complex. Inorg. Chem. 2017, 56, 1478-1482.

(40) Bhowmick, I.; Roehl, A. J.; Neilson, J. R.; Rappé, A. K.; Shores, M. P. Slow Magnetic Relaxation in Octahedral Low-Spin Ni(III) Complexes. Chem. Sci. 2018, 9, 6564-6571.

(41) Shrivastava, K. N. Theory of Spin-Lattice Ralaxation. Phys. Status Solidi B 1983, 117, 437-458.

(42) Tesi, L.; Lucaccini, E.; Cimatti, I.; Perfetti, M.; Mannini, M.; Atzori, M.; Morra, E.; Chiesa, M.; Caneschi, A.; Sorace, L.; Sessoli, R. Quantum Coherence in a Processable Vanadyl Complex: New Tools for the Search of Molecular Spin Qubits. Chem. Sci. 2016, 7, 20742083.

(43) Schweiger, A.; Jeschke, G. Chapter 8: Relaxation and Related Phenomena. Principles of Pulse Electron Paramagnetic Resonance; Oxford University Press, 2001; pp 208-233.

(44) Bader, K.; Winkler, M.; van Slageren, J. Tuning of Molecular Qubits: Very Long Coherence and Spin-Lattice Relaxation Times. Chem. Commun. 2016, 52, 3623-3626.

(45) Takahashi, S.; van Tol, J.; Beedle, C. C.; Hendrickson, D. N.; Brunel, L.-C.; Sherwin, M. S. Coherent Manipulation and 
Decoherence of $S=10$ Single-Molecule Magnets. Phys. Rev. Lett. 2009, 102, 087603.

(46) Pedersen, K. S.; Ariciu, A.-M.; McAdams, S.; Weihe, H.; Bendix, J.; Tuna, F.; Piligkos, S. Toward Molecular 4f Single-Ion Magnet Qubits. J. Am. Chem. Soc. 2016, 138, 5801-5804.

(47) Yamabayashi, T.; Atzori, M.; Tesi, L.; Cosquer, G.; Santanni, F.; Boulon, M.-E.; Morra, E.; Benci, S.; Torre, R.; Chiesa, M.; Sorace, L.; Sessoli, R.; Yamashita, M. Scaling Up Electronic Spin Qubits into a Three-Dimensional Metal-Organic Framework. J. Am. Chem. Soc. 2018, 140, 12090-12101.

(48) Zadrozny, J. M.; Niklas, J.; Poluektov, O. G.; Freedman, D. E. Millisecond Coherence Time in a Tunable Molecular Electronic Spin Qubit. ACS Cent. Sci. 2015, 1, 488-492.

(49) Atzori, M.; Chiesa, A.; Morra, E.; Chiesa, M.; Sorace, L.; Carretta, S.; Sessoli, R. A Two-Qubit Molecular Architecture for Electron-Mediated Nuclear Quantum Simulation. Chem. Sci. 2018, 9, 6183-6192.

(50) Shiddiq, M.; Komijani, D.; Duan, Y.; Gaita-Ariño, A.; Coronado, E.; Hill, S. Enhancing Coherence in Molecular Spin Qubits via Atomic Clock Transitions. Nature 2016, 531, 348-351.

(51) Baibekov, E. I.; Gafurov, M. R.; Zverev, D. G.; Kurkin, I. N.; Rodionov, A. A.; Malkin, B. Z.; Barbara, B. Coherent Spin Dynamics in a Gadolinium-Doped $\mathrm{CaWO}_{4}$ Crystal. Phys. Rev. B: Condens. Matter Mater. Phys. 2017, 95, 064427.

(52) Zadrozny, J. M.; Niklas, J.; Poluektov, O. G.; Freedman, D. E. Millisecond Coherence Time in a Tunable Molecular Electronic Spin Qubit. ACS Cent. Sci. 2015, 1, 488-492.

(53) Bader, K.; Dengler, D.; Lenz, S.; Endeward, B.; Jiang, S.-D.; Neugebauer, P.; van Slageren, J. Room Temperature Quantum Coherence in a Potential Molecular Qubit. Nat. Commun. 2014, 5, 5304.

(54) Rosaleny, L. E.; Cardona-Serra, S.; Escalera-Moreno, L.; Baldoví, J. J.; Gołębiewska, V.; Wlazło, K.; Casino, P.; Prima-García, H.; Gaita-Ariño, A.; Coronado, E. Peptides as Versatile Platforms for Quantum Computing. J. Phys. Chem. Lett. 2018, 9, 4522-4526.

(55) Graham, M. J.; Zadrozny, J. M.; Shiddiq, M.; Anderson, J. S.; Fataftah, M. S.; Hill, S.; Freedman, D. E. Influence of Electronic Spin and Spin-Orbit Coupling on Decoherence in Mononuclear Transition Metal Complexes. J. Am. Chem. Soc. 2014, 136, 7623-7626.

(56) Zadrozny, J. M.; Freedman, D. E. Qubit Control Limited by Spin-Lattice Relaxation in a Nuclear Spin-Free Iron(III) Complex. Inorg. Chem. 2015, 54, 12027-12031.

(57) Bertaina, S.; Gambarelli, S.; Tkachuk, A.; Kurkin, I. N.; Malkin, B.; Stepanov, A.; Barbara, B. Rare-Earth Solid-State Qubits. Nat. Nanotechnol. 2007, 2, 39-42.

(58) Naaman, R.; Waldeck, D. H. Chiral-Induced Spin Selectivity Effect. J. Phys. Chem. Lett. 2012, 3, 2178-2187. 